\title{
Interactive Storytelling with Literary Feelings
}

\author{
David Pizzi ${ }^{1}$, Fred Charles ${ }^{1}$, Jean-Luc Lugrin ${ }^{1}$ and Marc Cavazza ${ }^{1}$ \\ ${ }^{1}$ School of Computing, University of Teesside TS1 3BA, United Kingdom \\ \{d.pizzi, f.charles, j-1.lugrin, m.o.cavazza\}@tees.ac.uk
}

\begin{abstract}
In this paper, we describe the integration of Natural Language Processing (NLP) within an emotional planner to support Interactive Storytelling. Our emotional planner is based on a standard HSP planner, whose originality is drawn from altering the agents' beliefs and emotional states. Each character is driven by its own planner, while characters are able to operate on their reciprocal feelings thus affecting each other. Our baseline story is constituted by a classic XIX ${ }^{\text {th }}$ century French novel from Gustave Flaubert in which characters feelings play a dominant role. This approach benefits from the fact that Flaubert has described a specific ontology for his characters feelings. The objective of NLP should be to uncover from natural language utterances the same kind of affective elements, which requires an integration between NLP and the planning component at the level of semantic content. This research is illustrated with examples from a first fully integrated prototype comprising NLP, emotional planning and real-time 3D animation.
\end{abstract}

Keywords: Aesthetic computing, literary analysis, interactive storytelling, emotional NLP.

\section{Introduction}

Narratives traditionally depict emotions and feelings, and are similarly meant to elicit comparable ones in the mind of their readers (or spectators). This is why this issue has been addressed by research in Interactive Storytelling (IS). For instance, Cheong and Young have described how suspense could be elicited from formal properties of a sequence of narrative actions [11]. Previous research in IS has mostly addressed Ekmanian feelings, in particular fear [1] [15]. Fear is traditionally important as part of narratives: it can be depicted as experienced by characters and can also be elicited in the spectator, which contributes to realism as well as empathy.

However, studies of narrative aesthetics tend to uncover more sophisticated feelings. A study on a corpus of French novels has for instance uncovered over 128 such feelings [27] such as ambition, complicity, gratitude, guilt, loneliness, pride, shame, tact... Of course, literary feelings have no claim to psychological validity: they constitute a fine-grained semantics, which is very much related to aesthetic properties of the narrative. Their description is connected to other current topics in AI such as aesthetic computing [23], in which AI techniques are confronted with the processing of sophisticated cultural content, as in IS or the computational analysis of literary texts [3]. The distinction between psychological and literary feelings is another 
example of the duality between cognitive and semiotic approaches to cultural content, which, in the specific case of storytelling has been revisited by Christian and Young [12].

The majority of IS research prototypes have embraced planning as their core technology to control the behaviour of virtual actors, and drive the IS. In addition, the necessity to relate plan progression to dramatic elements has led to the adoption of emotional planning approaches, which will be discussed in the next section.

\subsection{System Overview and Architecture}

Our experimental system presents itself as a real-time 3D computer animation featuring virtual actors, which express themselves via animation and speech synthesis. The baseline story is adapted from a XIX ${ }^{\text {th }}$ century novel, Madame Bovary by Flaubert [13]. Only a small fragment of the original novel has been represented, more specifically chapters 9-12 of Part II [13]. The IS engine is based on a multi-threaded planner controlling each character independently. 3D animations are generated by the graphics engine from the grounded actions produced by the planner. During story visualisation, the system accepts NL input through the keyboard, which is analysed to update characters' beliefs and emotional state (i.e. by modifying character's mental states). In this way, the user interacts with the evolution of the narrative by influencing the selection of the next narrative action, albeit indirectly.

From a system architecture perspective (Figure 1), visualisation is provided by the Unreal Tournament ${ }^{\mathrm{TM}}$ game engine with which the planner communicates via UDP sockets. The communication between the planner and the visualisation engine takes place in both directions to acknowledge execution of actions in the virtual world by returning the resulting value (i.e. action success or failure). The planner itself is a $\mathrm{C}++$ implementation of the Heuristic Search Planner (HSP) approach (described in the next section) [4] [5]. The NLP module consists of an integrated parser (syntax and semantics) and is developed in Allegro Common Lisp: it communicates with the planner only, to which it sends new facts corresponding to the semantic interpretation of the utterance, also via a UDP socket.

With respect to previously described IS systems, this prototype relies on a baseline plot from an actual novel thus providing a realistic framework to explore the nature and consistency of interaction-driven alternatives.

\section{Emotional Planning for Interactive Storytelling}

The majority of IS research prototypes have relied on planning techniques for their narrative engine, generating action sequences as the story backbone [6] [24] [29]. Among the factors having favoured the adoption of planning was a tendency to present narratives as some kind of problem solving which is typical in epics, tales and certain genres such as the crime novel (e.g., plans to commit crimes such as robbery [30]).

The inclusion of emotions in IS systems has been extensively described by Gratch et al. [15] [17] and follows the development of emotional planning by Gratch [16]. 
They introduced the two central notions of appraisal (evaluating the emotional significance of events) and coping (maintaining the relationship between the agent and its social environment). However, in their approach emotions derive from an anticipation of plan evolution, for instance the anticipation of plan failure due to possible threats. In that sense, emotions do not belong to the planning domain itself.

If we now consider different narrative genres, emotions and feelings can actually be central to the plot itself and determine characters situation at every stage. We thus wanted to explore another approach, which consists in i) basing the plan domain itself on characters' feelings and ii) adopting a weaker representational model, in which planning is only used to drive action selection towards certain long-term objectives (rather than equating the story itself with a plan). Our planning component is based on a standard HSP approach, which provides additional flexibility in the generation of action sequences. The planning domain is composed of a list of characters' emotions and mental states characteristic of the novel. Some are represented on Figure 1, e.g.

reputation, embarrassment, loneliness.

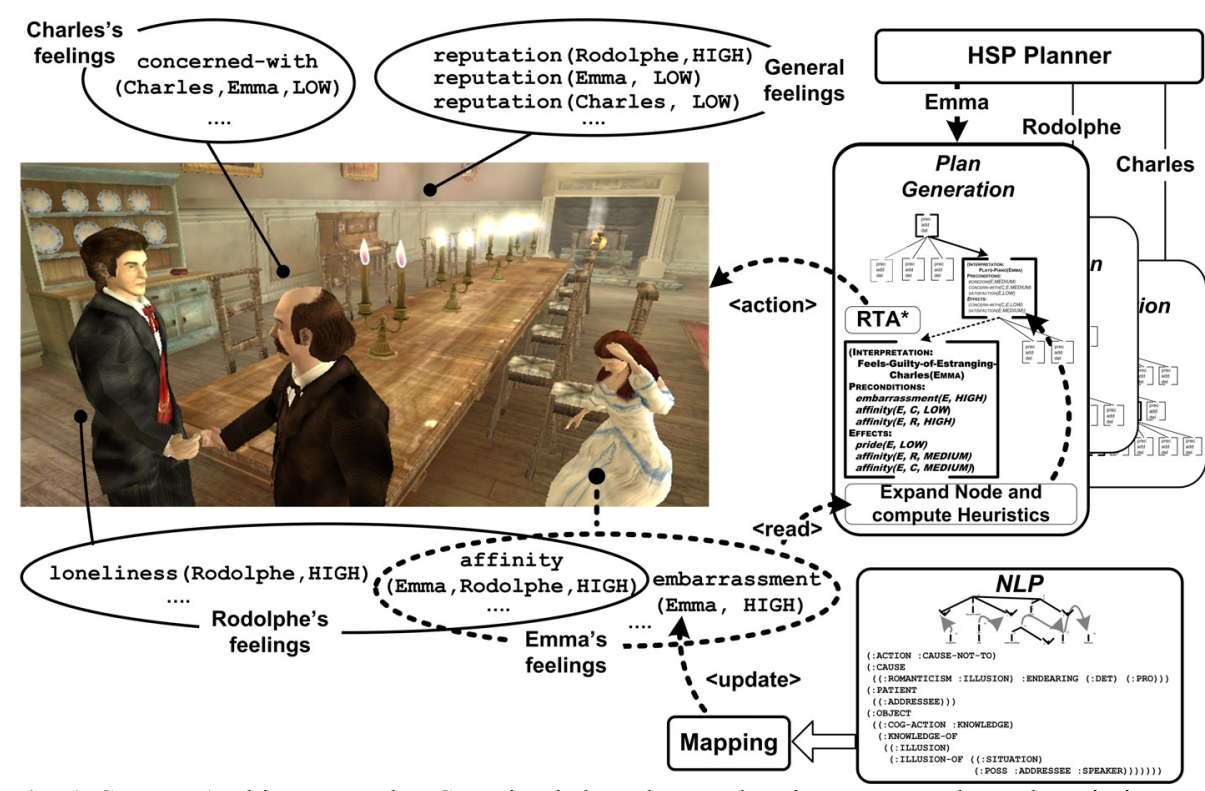

Fig. 1. System Architecture. The IS engine is based on a planning system whose domain is described in terms of characters' feelings. Characters can be influenced via Natural Language input.

These states admit various intensity values $\{\mathrm{LOW}, \mathrm{MEDIUM}, \mathrm{HIGH}\}$, which are modified by planning operators, themselves corresponding to feelings, which govern the evolution of mental states. These operators have been classified in three categories. Interpretation operators update a character's feelings to respond to a change in the state of the world, as with the following Disappointed-in or RegretsFalling-for (this being prompted by another character's actions or by the provision of new information, e.g. through user interaction). Figure 2 shows such an operator (Emboldened-by-love), which corresponds to a feeling of increased self-confidence 
for Emma Bovary. Character interaction operators intentionally modify another character's mental states and correspond to narrative actions such as invitations, arguments, and declarations (e.g. Accepts-Conversation, Makes-Love-Declaration, etc.). Finally, physical operators correspond to necessary physical actions such as changing location to interact with another character (the low-level details of motion planning and animation are automatically generated in the 3D environment from the corresponding action primitive). From the above description, it appears that the planning environment, in which each character influences other characters' feelings, is naturally dynamic, even more so considering the potential for user intervention at anytime. This is why we have opted for a "real-time" version of HSP by implementing RTA* [19] as its underlying search algorithm. Our implementation generates the next operator to be executed for each character, thus searching at a depth of one, without using look-ahead, and with a computational threshold of 500 ms. Finally, we use the simple Value Iteration (VI) method as described by Liu et al. [22] to calculate the heuristic function from the content of operators and the goal ("driver") definition. There is potential for further optimisation, for instance by using more sophisticated methods to calculate the heuristic function such as PINCH [22].

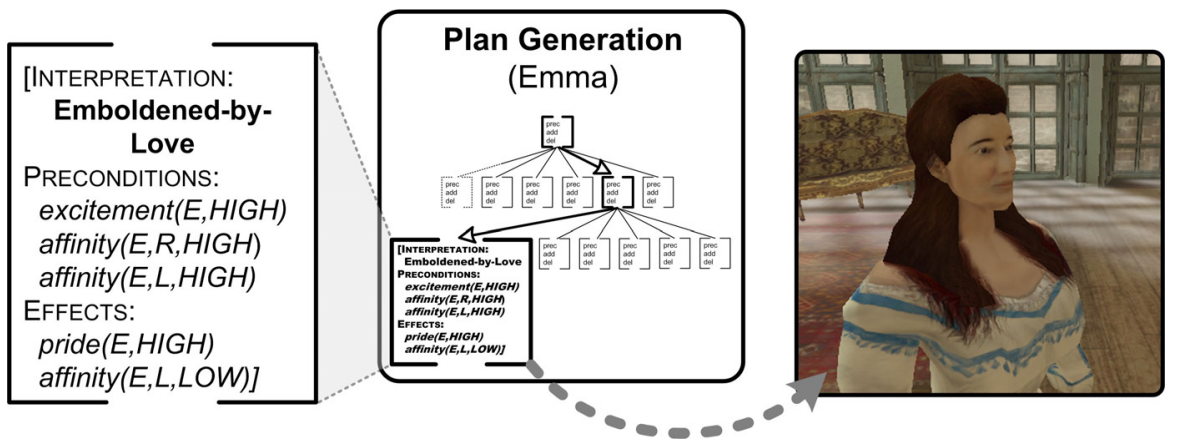

Fig. 2. An example Interpretation Operator: its contents are mental states and feelings relating the various characters $(\mathrm{E}=\mathrm{Emma}, \mathrm{L}=\mathrm{Lé}$ on, $\mathrm{R}=$ Rodolphe $)$.

\section{Narrative Formalisation: from Feelings to Planning Domains}

Character psychology and feelings permeate traditional forms of narratives such as $\mathrm{XIX}^{\text {th }}$ century novels. Gustave Flaubert's 1856 classic novel Madame Bovary is such an example. Further to placing significant emphasis on the characters' psychology, Flaubert's preliminary studies for the novel contain a description of the plot in the form of elementary plans and scenarios, together with an extensive description of the characters' psychology at various stages of the plot [21]. This description is actually based on his inventory of characters' feelings, accurate enough to constitute the basis for an "ontology".

Examples of such feelings described by Flaubert for Emma Bovary include feelingof-emptiness, boredom [21, p.17]; pride-of-having-a-lover, poetic-feelings [21, p.48] 
emboldened-by-love (developing an attitude), jealousy-curiosity [21, p. 48]; feelshatred-for-Charles [21, p. 49]; irritated-by-vice [21, p.50]; bitter-love-feelings [21, p.50]. Over 30 such feelings have been described by Flaubert in his preliminary plans and scenarios. This is a rather unique case of content formalisation provided by an author himself: it constitutes a formidable starting point for the design of an emotional planner, whose objective is to operate on the characters' feelings and mental states. These feelings range from traditional ones (e.g. boredom) to fairly specific ones. In deriving a planning domain from their inventory, we have first defined a set of ground mental states (such as affinity between characters, pride, womanhood), which were derived from the traditional feelings. Whereas the more specific feelings have been associated with Interpretation operators, which actually express the consequences of that literary feeling, and decompose it into ground mental states within the expression of the operator (both pre-conditions and effects).

Table 1. The definition of emotional (Interpretation) operators for virtual actors following the ontology feelings identified by Flaubert himself for Madame Bovary.

\begin{tabular}{|c|c|c|}
\hline $\begin{array}{c}\text { Feelings from Flaubert } \\
([21])\end{array}$ & Description & Operator \\
\hline $\begin{array}{l}\text { Pride-of-Having-a-Lover } \\
\text { (p. 48) }\end{array}$ & $\begin{array}{l}\text { The relationship between Emma } \\
\text { and Rodolphe is strong, and she } \\
\text { fulfils her dreams of living a } \\
\text { passionate love. }\end{array}$ & $\begin{array}{l}\text { [Interpretation: } \\
\text { Joy-of-Love (Emma) } \\
\text { Preconditions: } \\
\text { affinity(E, R, HIGH) } \\
\text { pride(E, HIGH) } \\
\text { womanhood(E, HIGH) } \\
\text { Effects: } \\
\text { satisfaction(E, HIGH)] }\end{array}$ \\
\hline $\begin{array}{l}\text { Irritated-by-Vice } \\
\text { (p. 50) }\end{array}$ & $\begin{array}{l}\text { Emma has doubts about their } \\
\text { relationship prompted by a letter } \\
\text { from her father enquiring about } \\
\text { her well-being. } \\
\text { At the same time, Rodolphe } \\
\text { behaviour is increasingly rude and } \\
\text { Emma is suffering from it. }\end{array}$ & $\begin{array}{l}\text { [Interpretation: } \\
\text { Regrets-Falling-for- } \\
\text { Rodolphe (Emma) } \\
\text { Preconditions: } \\
\text { embarrassment (E, HIGH) } \\
\text { affinity(E, C, MEDIUM) } \\
\text { power-over(R, E, HIGH) } \\
\text { Effects: } \\
\text { affinity (E, R, LOW) } \\
\text { anger(E, } R, H I G H) \\
\text { affinity(E, C, HIGH)] }\end{array}$ \\
\hline
\end{tabular}

This "ontology" plays a central role in the integration of AI technologies in our system, as it will be used to specify not only the planning domain but also the main semantic domains targeted by NL interpretation, as described in the next sections. When considering Flaubert's descriptions, there appears to be some kind of continuity between elementary mental states such as anger or pride, and the more sophisticated feelings of the type illustrated by Table I, which we have precisely termed "literary feelings". While Flaubert in his description does not explicitly formalise complex feelings in terms of elementary ones, this is an additional step we had to take to make the whole approach computational: this explains the above formalisations, in which mental states tend to feature as fluents belonging to operators' preconditions, while operators themselves have been associated to literary feelings. 


\section{Emotional NLP for Interactive Storytelling}

The ability to incorporate Natural Language in IS remains a major challenge that has received comparatively less attention than other aspects such as action generation. Language is an important element of the aesthetics of narratives: in the case of IS it plays a dual role by being both part of the staged drama, and the privileged modality for user interaction. For that reason, and from the long-term perspective of explicitly addressing literary feelings in IS, it is important to lay some foundations for its investigation.

Previous IS systems having incorporated NLP (under written [25] or spoken [6] [31] form) can be divided into two categories depending on whether user utterances are actually meant to form part of the staged IS or not. In the latter case [6], they support intervention from a spectator who influences the characters from a God-like perspective: as such, they follow normal user expressions and are unrelated to narrative aesthetics. However, the most challenging case consists of utterances, which, because they should also be part of the (interactive) narrative's dialogues, have to contribute to the aesthetics of the narrative. Emotional NLP in the context of these aesthetic expressions is thus faced with the double challenge of parsing complexity, because the style will often be literary rather than common, and the extraction of implicit or figurative meaning, which will ultimately have to be interpreted in terms of characters' feelings. We have used sentences derived from the English translation of Madame Bovary to experiment with NL input. Despite variations in style in the translations [32] they presented similar characteristics in terms of style and implicit meaning, including emotional aspects.

To a large extent, it can be said that the whole objective of the NLP step is to uncover feelings from the semantic content of the utterance, which are part of the planners' domain. To address this challenge, we propose to direct the whole NLP step towards the identification of specific feelings by using a highly contextual approach to the lexicon directly inspired from Textual Semantics [28].

Textual Semantics posits that the relevant semantic content of the lexicon actually corresponds to highly contextual categories rather than generic ones (this differs from the introduction of generic emotional tags [33] and corresponds to specific types of applications). This leads to a redefinition of lexical content in context, based on the identification of the most relevant semantic domains. In the case of Madame Bovary, one can identify dimensions such as /boredom/ vs. /enjoyment/, /illusion/ vs. /reality/, /fear-for-reputation/ vs. /acceptance-of-risk/, etc. Rastier [27] has in particular demonstrated that the concept of /boredom/, whilst central to Madame Bovary was however not lexicalised in the novel's text (only 4 occurrences in the whole of the original text) and constituted instead a semantic category into which a variety of words would be indexed. This encourages us to identify semantic categories corresponding to the novel main topics (as in the above semantic oppositions).

The potential of this approach can be compared to previous work on NLP in IS. It has been generally based on the identification of dialogue acts which were then mapped to specific narrative functions (or in certain cases even equated to them). The number of speech acts varies but can be reduced to evaluative dimensions such as agree/disagree [9] [25]. 
Let us consider the utterance [13]:

"And I shall remain tonight, tomorrow, all other days, all my life!" (S1)

A traditional speech act interpretation would categorise this utterance as a "promise" and more specifically in the romantic context of the novel, an "eternal love promise". This would assume a corresponding narrative function in the IS system, but the main difficulty would be to identify such a speech act from either the surface form or the semantic content. However, it can also be interpreted in terms of semantic features such as /lover-presence/ and/duration/, which can then be mapped directly to the planning domain. The principle behind that mapping, which follows from early work described in [7] consists in identifying feelings as collections of semantic features [27]. Similar principles have been described more recently: by Basili and Marocco [2] using co-occurrence analysis and Latent Semantic Analysis [20] and by Gliozzo [14] using semantic domains, which are equivalent to the semantic classes of Textual Semantics [28].

We have attempted a first small-scale implementation of these principles. The linguistic coverage is both limited and specific to certain constructs encountered in our sample corpus. However the principles aim at being generic (the only large-scale NLP system used in IS has been described by Mateas [26] as "author-intensive", suggesting a strong intertwining of linguistic and narrative representations, probably justified by the fact that the Façade narrative is essentially dialogue-based). Our NLP component aims at constructing a feature structure based on (contextual) semantic features associated to the lexicon.

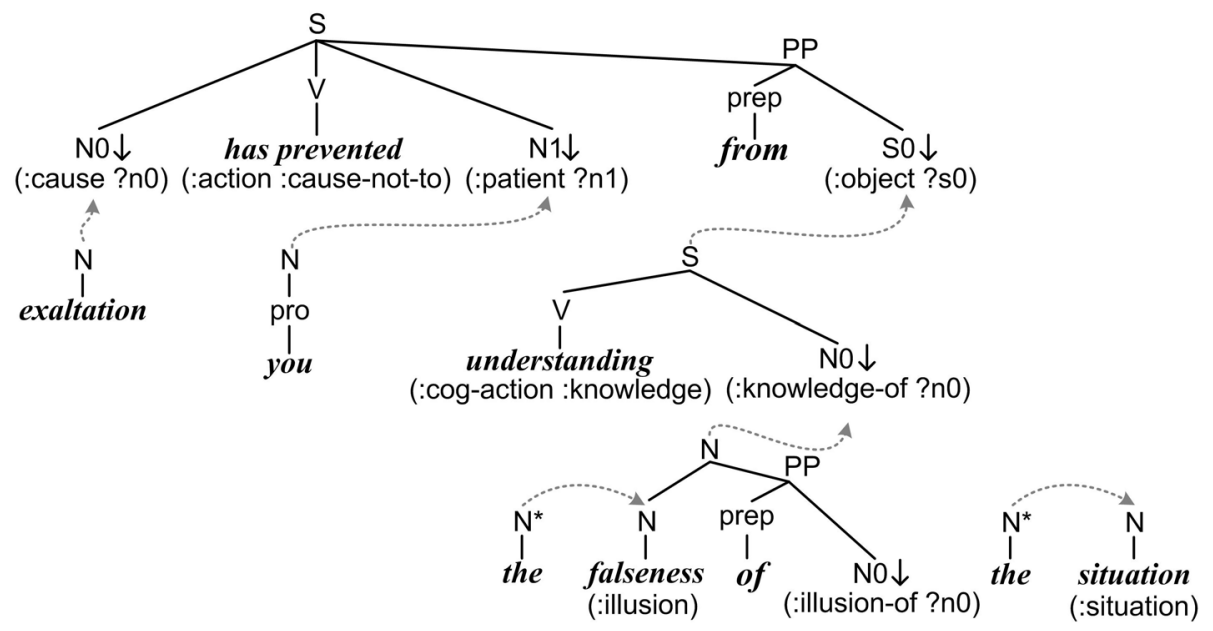

Fig. 3. Partial representation of the parsing of an actual sentence from the novel with a lexicalised grammar.

The construction of such a semantic structure is driven by syntactic analysis. We have developed an ad-hoc lexicalised grammar covering some major syntactic forms encountered in the set of dialogues and monologues occurring in the novel. This lexicalised grammar is derived from a simplified version of the Tree-Adjoining Grammar (TAG) [18] formalism in which adjunction is replaced with the operation of 
furcation [8]. The use of TAG also facilitates the processing of idiomatic constructs, which are frequent in that literary genre. The parser we used is adapted from an earlier implementation [8], which integrates syntactic and semantic processing, as its main purpose is to construct a semantic structure. Parsing proceeds bottom up, as adjacent trees of compatible types are combined through substitution and furcation. During furcation, semantic features are propagated from the auxiliary to the target tree to progressively build up a semantic structure (Figure 3). Substitution establishes semantic relations between the related trees, creating a nested feature structure. In those cases where the parser is unable to produce a complete parse, the semantic structures obtained from the various partial parses are merged into a single structure. In other words, parsing is not about identifying named entities or even uncovering (narrative) action structure but establishing the recurrence of semantic features associated to feelings.

The Emotional NLP module operates in three steps: i) the TAG parser assembles a semantic structure whilst processing the sentence. In case no single parse of head $\mathrm{S}$ can be produced, the semantic structures for partial parses are merged into one; ii) this semantic structure is interpreted, not as a logical formula or as a case structure, but as a resource for the extraction of semantic patterns based on emotional categories (feelings) for the novel and; iii) these semantic patterns are mapped to the planning domain using association rules. Certain patterns can actually take part in the preconditions of certain operators: for instance an inventory of properties (illusion, selfishness, prodigality) can be mapped to certain key feelings such as embarrassment when brought to the attention of Emma's consciousness.

To illustrate this let us consider the analysis of the following sentence:

"This charming exaltation has prevented you from understanding the falseness of our future situation" (S2)

The complexity of this sentence in terms of interpretation makes it challenging for traditional NLP techniques to extract an appropriate (implicit) meaning and map it subsequently to the planning domain. However, if we assign as a goal to the parser to construct an integrated semantic feature structure, it can support the identification of feature patterns that map onto feelings in the planning domain.

Part of the parsing process is represented on Figure 3 and the resulting feature structure on Figure 4.

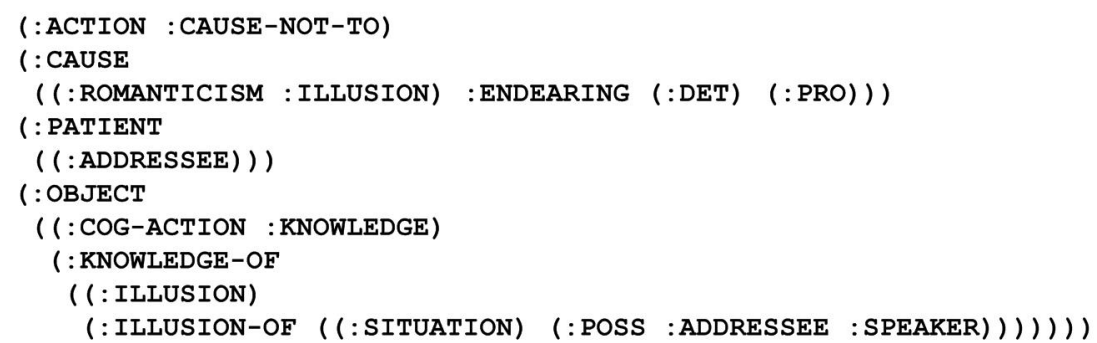

Fig. 4. Semantics Structure obtained for sentence 2.

Its analysis results in a fairly complex and redundant semantic structure formed by the aggregation of features and establishment of case relations (Figure 4). The central point is how semantic description through a very focussed set of features 
corresponding to the novel's main dimensions (:illusion, :illusion-of) supports further interpretation which would otherwise be beyond reach. In the semantic structure, :speaker and :addressee are first replaced by Rodolphe and Emma. The key expression (:illusion-of ((:situation (:poss Emma Rodolphe)))) together with the recurrence of the :illusion feature can be mapped to domain facts such as embarrassment (Emma, HIGH). We have defined a small number of rules for that mapping, which embody the main narrative dimensions (as illustrated in section 2). Semantic features associated to lexical entries are described using differential semantics [28], i.e. by identifying salient features within semantic classes (equivalent to "Synsets", although with a stronger focus, which makes them "minimal" meaning classes [28]). These classes themselves derive from an identification of emotional vocabulary in French novels, which obviously apply to the current context [27].

\section{Prototype Integration and Example Results}

A first version of the prototype has been implemented comprising a total of 70 operators and 150 logical atoms in the Planner's domain. At its present stage of development, the system has not been the object of formalised evaluation which, in the general case of IS, remains a research topic in itself [10]. The system has however been tested for the generation of variants of the baseline plot from different initial conditions and is able to produce multiple stories of an average duration of 4 minutes.

Figure 5 illustrates the impact of an utterance on the story unfolding from the perspective of the central character, Emma Bovary. The initial situation corresponds to Emma and Rodolphe meeting just after she has decided to reach happiness (which is translated in the planning domain by adding a new local goal represented by the feeling satisfaction(E, HIGH)). Emma is estranging her husband Charles (Disappointed-in-Charles), after he fell into disrepute due to medical malpractice, and she is ready to take any risk (accepts-adultery-risk ( $E, C, H I G H)$ ) to escape from a life she sees as miserable. In absence of influence (left hand side of the figure), Emma will fulfil her goal by letting Rodolphe seduce her. She engages first in a romantic conversation (Accepts-Conversation-Rodolphe and Say-Sthg-inConfidence-Rodolphe) before falling for her new lover and showing him affection signs (Kissed-by-Rodolphe, Physical-Contact-with-Rodolphe). She is now living in a new relationship and starts to enjoy it (In-Love-Attitude and Emboldened-byLove), which will make her forget about Leon (her ex-lover who moved away). At the end, she will not hesitate to risk her financial situation for him (offer-Gifts-toRodolphe) before at last being proud of this new lover (Joy-of-Love). This to a large extent corresponds to a generation of the original storyline by the system, which would be a first indication that the planning domain is consistent.

Conversely, the right-hand side of the figure shows alternative story evolutions from exactly the same initial conditions, under the influence of a Natural Language utterance introduced at different stages. This utterance is entered by the user on behalf of Rodolphe ( $S 2$ or "you should not be one of those frivolous women"). This type of criticism is most likely to generate Embarrassment. However, depending on the stage at which this utterance is introduced, Emma will react differently. At an early stage, where her feelings for Rodolphe are not that strong (marked as A and B of Figure 5), 
she will soon return to her family (Emphasises-Motherhood and Joy-of-Family), eventually feeling compassion for Charles (Commiseration). However, at a more advanced stage of her relation (Figure $5 \mathrm{C}$ ), following her first affection signs to Rodolphe (Kissed-by-Rodolphe, Physical-Contact-with-Rodolphe), Emma will experience feelings of guilt (Feels-Guilty-of-Estranging-Charles) and will hesitate between her husband and her lover (Love-Ambivalence). In absence of new seduction acts from Rodolphe, she will also return to her family (EmphasisesMotherhood and Joy-of-Family). The same utterance at a later stage of their relation (Figure 5 D), will upset Emma about the power that actually Rodolphe has gained over her (Regrets-Falling-for-Rodolphe), prompting her to return to her family, more as a rejection of Rodolphe.

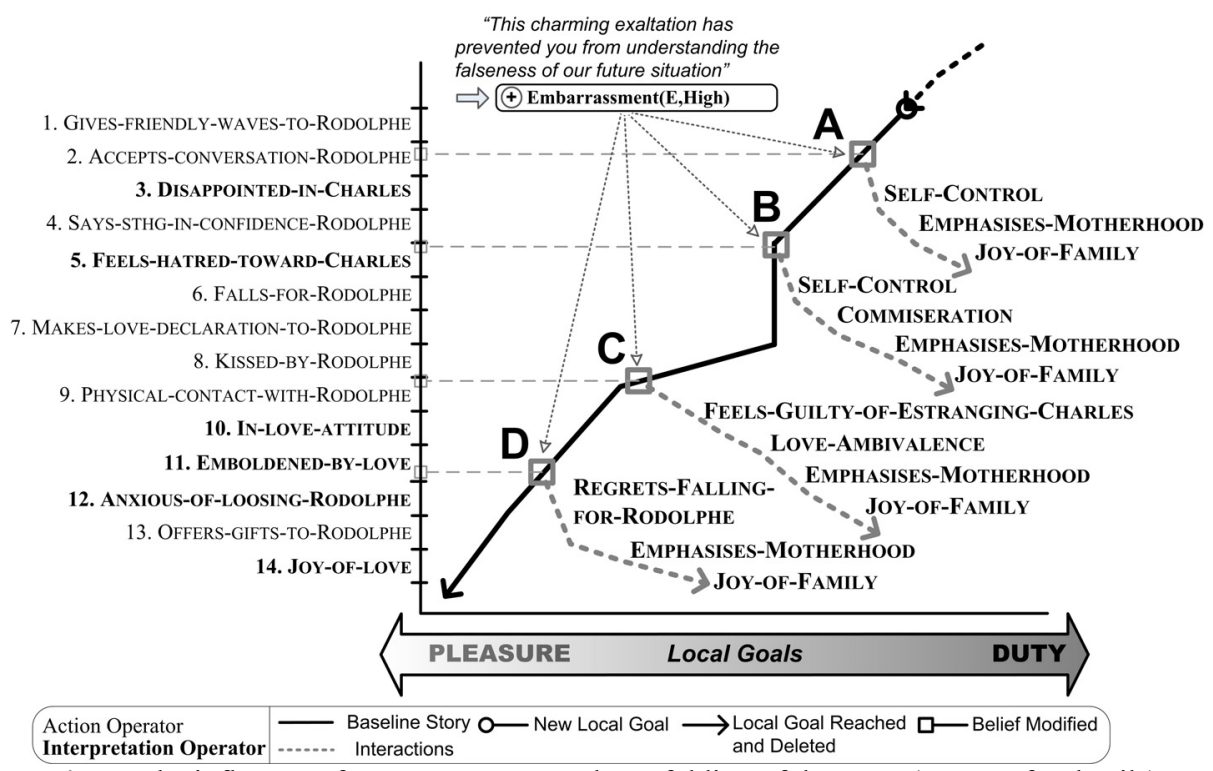

Fig. 5. The influence of a NL utterance on the unfolding of the story (see text for details).

If however this utterance takes place once their affair has transformed Emma's personality (Emboldened-by-Love), it will have no effect on Emma's subsequent behaviour, as she will reach happiness through this new love (Joy-of-Love).

\section{Conclusions}

We have presented an approach to IS based on characters' feelings and the use of such feelings to implement the planning domain of the IS engine. This integration has emphasised content and knowledge representation aspects, as can be expected for an application processing aesthetic content. While this is largely a research prototype of moderate scale, it could form the basis for new IS technologies. In particular a more flexible interpretation of NL input could circumvent the inevitable limitations of parsing for utterances extracted from literary texts, as well as the difficulties of 
specific speech acts identification, thereby facilitating integration with storytelling engines based on planning technologies. This approach falls under the generic distinction between the cognitive and the narrative (See also [12] for a specific discussion in the context of IS).

Acknowledgments. This work has been funded in part by the Department of Trade and Industry, via the Technology Programme BARDS Project, in collaboration with Eidos Interactive Ltd.

\section{References}

1. Aylett, R., Louchart, S., Dias, J., Paiva, A., Vala, M.: FearNot! - An Experiment in Emergent Narrative. In: Panayiotopoulos, T., Gratch, J., Aylett, R., Ballin, D., Olivierand, P., Rist, T., (Eds.): Intelligent Virtual Agents, $5^{\text {th }}$ International Working Conference (2005) 305-316.

2. Basili, R., Marocco, P.: A geometrical approach to literary text analysis, Proceedings of the LREC 2006 Workshop "Towards Computational models of Literary Analysis", Genova, Italy, May 22nd (2006).

3. Basili, R.: Toward Computational Models of Literary Analysis, (Ed.), Proceedings of the LREC 2006 workshop, Genova, Italy (2006).

4. Bonet, B., Geffner, H.: Planning as Heuristic Search: New Results, Proceedings of the European Conference on Planning, ECP'99 (1999) 360-372.

5. Bonet, B., Geffner, H.: Planning as Heuristic Search. Artificial Intelligence Special Issue on Heuristic Search, 129, n.1. (2001) 5-33.

6. Cavazza, M., Charles, F., Mead, S.J.: Interacting with Virtual Characters in Interactive Storytelling. Proceedings of the First International Joint Conference on Autonomous Agents and Multi-Agent Systems, AAMAS'02, Bologna, Italy (2002) 318-325.

7. Cavazza, M., Martin, O., Charles, F., Mead, S.J. and Marichal, X.: Interacting with Virtual Agents in Interactive Storytelling. International Conference on Intelligent Virtual Agents (2003).

8. Cavazza, M.: An Integrated Parser for TFG with Explicit Tree Typing. Proceedings of the Fourth TAG+ Workshop, University of Pennsylvania (1998).

9. Cavazza, M., Martin, O., Charles, F., Mead, S.J., Marichal, X., Nandi, A.: Multi-modal Acting in Mixed Reality Interactive Storytelling. IEEE Multimedia, July-September 2004, Vol. 11, Issue 3 (2004).

10. Charles, F., Cavazza, M.: Exploring the Scalability of Character-based Storytelling. In 3rd International Joint Conference on Autonomous Agents and Multi-Agent Systems, AAMAS'2004, New York, ACM Press (2004) 872-879.

11. Cheong, Y.-G., Young, R. M.: A Computational Model of Narrative Generation for Suspense. In the AAAI 2006 Workshop on Computational Aesthetics (2006).

12. Christian, D.B., Young, R. M.: Comparing Cognitive and Computational Models of Narrative Structure. Proceedings of the Nineteenth National Conference on Artificial Intelligence, AAAI 2004 (2004) 385-390.

13. Flaubert, G.: Madame Bovary. La revue de Paris (Ed.), France, (1856) (in French).

14. Gliozzo, A.M.: Semantic Domains and Linguistic Theory. In Proceedings of the LREC 2006 workshop "Toward Computational Models of Literary Analysis", Genova, Italy (2006). 
15. Gratch, J., Marsella, S.: Tears and fears: Modeling emotions and emotional behaviors in synthetic agents. In Proceedings of the Fifth International Conference on Autonomous Agents (2001) 278-285.

16. Gratch, J.: Why you should buy an emotional planner. Proceedings of the Autonomous Agents, Workshop on Emotion-based Agent Architectures , EBAA'99 (1999).

17. Gratch, J., Marsella, S., Mao, W.: Towards a Validated Model of "Emotional Intelligence". Twenty-First National Conference on Artificial Intelligence, AAAI06, Boston, MA (2006).

18. Joshi, A. K., Schabes, Y.: Tree-adjoining grammars and lexicalized grammars. In Maurice Nivat and Andreas Podelski, editors, Tree Automata and Languages, Elsevier Science (1992).

19. Korf, R.E.: Real-time heuristic search. Artificial Intelligence, 42:2-3, (1990) 189-211

20. Landauer, T. K., Dumais, S. T.: A solution to Plato's problem: the Latent Semantic Analysis theory of acquisition, induction and representation of knowledge. Psychological Review, 104(2) (1997) 211-240.

21. Leclerc, Y. (Ed.): Plans et Scenarios de Madame Bovary, CNRS Editions, France (1995) (in French).

22. Liu, Y., Koenig, S., Furcy, D.: Speeding Up the Calculation of Heuristics for Heuristic Search-Based Planning. In Proceedings of the Eighteenth National Conference on Artificial Intelligence, AAAI2002 (2002) 484-491.

23. Liu, H., Mihalcea, R.: Computational Aesthetics: Artificial Intelligence Approaches to Beauty and Happiness. AAAI Workshop, ed. Technical Report WS-06-04. American Association for Artificial Intelligence, Menlo Park, California (2006) 8-15.

24. Magerko, B., Laird, J.E., Assanie, M., Kerfoot, A., Stokes, D.: AI Characters and Directors for Interactive Computer Games. Proceedings of the Nineteenth National Conference on Artificial Intelligence (2004) 877-883.

25. Mateas, M., Stern, A.: Natural Language Understanding in Façade: Surface-Text Processing. 2nd International Conference on Technologies for Interactive Digital Storytelling and Entertainment, TIDSE 2004 (2004) 3-13.

26. Mehta, M., Dow, S., Mateas, M., MacIntyre, B.: Evaluating a Conversation-Centered Interactive drama. Proceedings of the Sixth International Conference on Autonomous Agents and Multiagent Systems, AAMAS'07, Honolulu, Hawaii, USA (2007).

27. Rastier, F.: La sémantique des thèmes - ou le voyage sentimental. In F. Rastier (Ed.), L'analyse thématique des données textuelles. L'exemple des sentiments, Paris: Didier (1995) 223-249 (in French).

28. Rastier, F., Cavazza, M., Abeille, A.: Semantics for Descriptions: From Linguistics to Computer Science. CSLI Lecture Notes 138, University of Chicago Press (2001).

29. Riedl, M.O., Young, R.M.: An Intent-Driven Planner for Multi-Agent Story Generation. Third ACM Joint Conference on Autonomous Agents and Multi-Agent Systems, AAMAS'04, New York, USA (2004) 186-193.

30. Riedl, M.O., Young, R.M.: From Linear Story Generation to Branching Story Graphs. IEEE Computer Graphics and Applications 26(3) (2006) 23-31.

31. Swartout, W., Gratch, J., Hill, R., Hovy, E., Marsella, S., Rickel, J., Traum, D.: Toward Virtual Humans, AI Magazine, 27(1) (2006).

32. Uzuner, Ö., Katz, B.: Capturing Expression Using Linguistic Information. Proceedings of the Twentieth National Conference on Artificial Intelligence, AAAI 2005 (2005) 11241130.

33. Valitutti, A., Strapparava, C., Stock, O.: Lexical Resources and Semantic Similarity for Affective Evaluative Expressions Generation, ACII 2005 (2005) 474-481. 